\title{
Harvesting the Benefits of Understanding Cancer with the Use of 'Shuttle' Era Technology- Cellular Survival Strategies and Tissue Engineering Via Grafting of Genetically Immortalized Autologous Cells
} John A. Loudon*

Wetherill Park Medical Centre,(Dr George Al-Horani, Principal) Suite 101, Stockland Mall,Polding Street, Wetherill Park,Sydney, NSW, 2164 Australia

\begin{abstract}
This review aims to focus on several of the advantages that an improved understanding of the biology of cancer may offer medicine. Following in the wake of my recent review demonstrating the close molecular mechanistic relationship between heart survival mechanisms and those of cancer under environmental challenge I examine how cancer can provide survival answers for tissues under physiological challenge. I continue the basis of this discussion with the argument that cancer can offer clues as to how to better develop reliable and sound strategies for tissue/organ regeneration. For a number of years the concept with the use of stem cells to enable tissue and/ or organ regeneration has gained attention. Indeed, recently, tooth buds have been regenerated from mouse stem cells allowing complete tooth regeneration in vivo. In this review, the focus is on alternative means for tissue/ organ regeneration by harnessing the all-sort-after property of cellular immortality that cancer possesses in its armamentarium. Literature is presented to demonstrate the utility and practicality of immortal genetic modification of autologous post-mitotic and senescent cells for reimplantation. This process may be of practical value in numerous medical situations from degenerative neurological diseases, wound healing, hair follicle replacement and auditory repair to aiding functional repair of cardiac disease to name but a few. The rational benefits of this technique are presented over stem cell technologies and potential technical obstacles are outlined that may need to be overcome. Novel technologies derived from the use of shuttle expression vectors are outlined here to screen for therapyresistance genes in cancer. These methods may be further enhanced and developed to allow delineation of genes involved in preventing cellular senescence whilst not transforming cells to a cancerous phenotype. These genes can subsequently be used in genetic modification of autologously harvested cells for re-implantation to enable tissue and/or organ regeneration.
\end{abstract}

Keywords: Human telomerase; Tumor suppressors; Oncogenes; p16; Immortal cell lines; Transfection; Shuttle vector; Proteomics; Wound healing; Tissue regeneration; Prosurvival model; Survival pathways

\section{Introduction}

A very interesting study reported this year [1] has outlined that by using a patient's own cells urethras can be made out of seeding those very same cells onto a scaffold of tubularised polyglycolic acid:poly(lactide-co-glycolide). Both autologously harvested muscle and epithelial cells were expanded and successfully re-implanted and provided functioning tissues for patients who required reconstruction. In fact, these artificial urethras worked well for the follow-up period of 6 years. An earlier much cited study showed that autologously harvested urothelial and muscle cells grown in culture and seeded on a bladder-shaped scaffold of collagen and/or polyglycolic acid wrapped in omentum and subsequently implanted demonstrated adequate function for individuals requiring cystoplasty [2].

Such work appears to usher in a new era of engineering for replacement of tissues and indeed defective organs. Certainly this avoids harvesting from alternative body sites and the inherent difficulties in harvesting from other individuals since allografts may suffer rejection. As a follow-on from these successful works I would like to propose support for the concept that takes this technology one step further and harness some of the intrinsically unique and powerful properties of cancer. This grew out of posing the question: why simply re-implant autologously harvested normal cells - why not improve upon them in respect to the purposes intended and then re-implant? This is the point I wish to address in this present review and expand upon. Firstly though, I should like to discuss some relevant background to this proposition.

Harvesting the benefits an understanding of cancer has to offer - cancer, the 'ultimate survivor'

To place this section in perspective, I myself am a cancer and cardiovascular research scientist and publish in those areas [3-6]. My support for the present concept arose whilst examining cancer literature regarding an earlier oral cancer project I have been involved in [7, see below]. Overall, my studies have been aimed at attempting to appreciate and indeed harvest the benefits that an understanding of cancer may yield. For example, in a recent article [5]. I indicated that the reader ought to be reminded that although cancer is an untamed parasite with regards its behavior it can be bridled as it were with the attendant advantages to medicine that that may offer. For example, monoclonal antibodies and transgenic-mediated oncogenesis technology stem

*Corresponding author: John A. Loudon Wetherill Park Medical Centre Suite 101, Stockland Mall,Polding Street, Wetherill Park,Sydney, NSW, 2164 Australia, Tel: +61297563636; Fax: +61297563637; E-mail: jon.uk1515@gmail.com

Received August 09, 2011; Accepted October 24, 2011; Published October 27 2011

Citation: Loudon JA (2011) Harvesting the Benefits of Understanding Cancer with the Use of 'Shuttle' Era Technology- Cellular Survival Strategies and Tissue Engineering Via Grafting of Genetically Immortalized Autologous Cells. J Bioanal Biomed S5: 001. doi:10.4172/1948-593X.S5-001

Copyright: @ 2011 Loudon JA. This is an open-access article distributed under the terms of the Creative Commons Attribution License, which permits unrestricted use, distribution, and reproduction in any medium, provided the original author and source are credited. 
Citation: Loudon JA (2011) Harvesting the Benefits of Understanding Cancer with the Use of 'Shuttle' Era Technology- Cellular Survival Strategies and Tissue Engineering Via Grafting of Genetically Immortalized Autologous Cells. J Bioanal Biomed S5: 001. doi:10.4172/1948-593X. S5-001

Page 2 of 10

from harnessing cancer's properties. I myself have benefited in my own research from the application of the latter methodology [3].

My further research has revealed that there are indeed many commonalities between cancer's survival mechanisms and those used by normal tissues - particularly under physiological challenge (6). This can be taken to some advantage in order to develop predictive systems and models for prosurvival mechanisms in many organs such as the heart [6], given that one may look at cancer as the 'ultimate survivor'. Indeed, as I have also discussed given this tight mutual relationship between cancer biology and normal tissue biology [6], heart survival mechanisms under environmental challenge may predict mechanisms used by cancer to cope with external challenge such as chemo/radiotherapy and in producing cancer dormancy - a reactive hibernating survival strategy oftentimes used by cancer.

As mentioned above, cancer itself provides an ideal model for cellular survival for physiological situations, for example, in cardio protection. In fact, as stated [6] according to this approach, cancer can be used to predict mechanisms and pathways used by organs and tissues to counteract external challenges. For example, cardio protective biomarkers can be predicted by cancer. These have been sought after for many years by cardiovascular researchers and the model I propose [6] will elucidate these factors using cancer as a model for cell survival. As my background originally comes from cardiovascular physiology originally, not in fact cancer, my interest lies in achieving this. Examples of cardioprotective factors that were found originally in cancer as survival factors and significantly predate that experimentally for the heart include peroxiredoxin [8]. Later on in 2006 [9] it was found that disruption of a peroxiredoxin family member ( $\operatorname{Prdx} 6)$, a peroxide scavenger, leads to heart vulnerability to ischaemia-reperfusion injury; that is, this factor is cardioprotective. In another example, Adriamycinresistant MCF-7 human mammary carcinoma cells, but not cells sensitive to this drug, express alpha4beta1 and alpha5betal integrins [10]. Curiously, it was later found that angiopoietin-1 promotes cardiac cell survival-cardioprotection-via integrins [11]. Therefore cancer studies well and truly predate those in the heart in terms of discovering that integrins have vital roles to play in cardioprotection.

Another key example is that of ER/PERK. Endoplasmic reticulum stress-response has been denoted as a "novel therapeutic target" in heart diseases [12]. Indeed, ER stress via such factors as PERK (eukaryotic translation initiator factor 2alpha kinase RNA-dependent protein kinase-like ER kinase) has been indicated of late in an autophagyrelated mechanism to lead to cardioprotection [13]. It comes as little surprise that in fact several years ago ER stress activation of $\mathrm{p} 38$ via PERK activation and BiP expression protects dormant cancer cells from chemotherapy [14]. PERK - dependent phosphorylation of eIF2alpha is one part of the Unfolded Protein Response (UPR) a coordinated cellular programme that leads to cell survival under ER challenge. Thus UPR is a target for anticancer therapies and indeed is a target for mediating cardioprotection too.

Many factors have been examined as cancer therapy-resistance related and ought to be tested in terms of their involvement in cardioprotection such as: Gp96 an ER resident heat shock protein and a cancer therapy resistance marker [15] Junction adhesion factors - claudin - are overexpressed in cisplatin resistant ovarian cancer cells as are other factors such as cell recognition molecule CASPR3, S100 family members and CDC42-binding protein kinase beta.
These are biomarkers of treatment response and as such treatment targets [16]. Interestingly, Claudin has not been looked at in terms of cardioprotection nor has CASPR3 nor CDC42-binding protein kinase beta. Nonetheless, S100 has been looked at in cardioprotection as a factor that assists mediation of ischemic preconditioning [17]. This observation is very much consistent with my prosurvival model [6].

Rho and Rho kinase are known to act in actin filament organization and it has been shown that Rho-Rho kinase pathway is involved in early ischemic cardioprotective preconditioning [18]. This study was from this year. Interestingly, in cancer under environmental challenge such as under irradiation the Rho-signaling pathway is needed for radiation resistance and induced invasion of glioblastoma cells [19]. Notably this study was performed 5 years ago in 2006 and points out that the Rho pathway is not needed for invasion under physiological conditions that is, nil external challenge. This 2006 investigation delineates Rho as a biomarker for targeting under external challenge. It comes as little surprise in terms of the 'pro-survival' model and also makes a further statement regarding the predictability of cancer external challenge survival paths to those of the heart (and indeed, more broadly, with respect to other organs too doubtlessly).

These works mentioned above speak of the potential of cancer, seen as an adapted and evolved 'true survivor' to predict mechanisms of survival under challenge for biological tissues generally.

Many other instances can be mentioned of where cancer particularly radiotherapy or chemotherapy resistant cancer - may demonstrate pathways leading to cardioprotection and predict same. Factors outlined as related to radiotherapy resistance in cancer [20] such as pre-mRNA-processing factor 19 (PRPF19) involved in DNA repair is protective of damage induced by environmental agents such as radiotherapy and chemotherapy and thus is prosurvival in cancer. Later analysis has shown that this factor is anti-apoptotic in heart [21] consistent with the overall proposed model.

Dihydropyrimidinase-related protein 2 (CRMP2) has been indicated to be a cancer biomarker [22] and a factor expressed in radioresistant cancer [20]. This factor has essentially not been looked at in heart - only to say that it is expressed [23]. The message here from cancer is to explore this factor as a cardioprotective biomarker.

All these data and more shall be the subject of a future cardiovascular-based review of mine. Nonetheless, the introduction I have provided to this relationship strengthens the notion of the tangible benefits understanding cancer may offer - which is a focus of this present review.

\section{The immortality principle}

Having said all of the above, the major feature and property of cancer that intrigues is its immortal nature. In culture, cancer cells may keep dividing indefinitely and do not naturally senesce. This property was particularly emphasized to me by a study of several years ago relating to oral cancer [7] as mentioned above. In this work, immortalization of an oral dysplastic epithelial cell line was produced with telomerase transfection in addition to loss of p16Ink4a and/or retinoic acid receptor (RAR) beta. Importantly p53 activity remained as wild type in the immortal clones.

Mortal-type dysplasias remain mortal even in the presence of hTERT (human telomerase reverse transcriptase) with elongation 
Citation: Loudon JA (2011) Harvesting the Benefits of Understanding Cancer with the Use of 'Shuttle' Era Technology- Cellular Survival Strategies and Tissue Engineering Via Grafting of Genetically Immortalized Autologous Cells. J Bioanal Biomed S5: 001. doi:10.4172/1948-593X. S5-001

Page 3 of 10

of telomeric structures if their p16Ink4A and RAR beta expression are maintained. Loss of p16Ink4A and RAR beta alone leads to an extended lifespan and delayed but ultimate senescence. It is crucial to investigate that in fact hTERT addition combined with p16 loss will lead to predictable cellular immortalization without development of the cancerous phenotype. This has indeed been borne out in the literature. Immortal ameloblastoma cells were induced by retroviral transfection with hTERT and the mechanism to immortality was via loss of p16 and not p21 nor p53 [24]. Importantly, these immortally produced cells did not undergo malignant transformation as assessed by the nudemouse tumorigenicity assay. This is of interest as ameloblastoma is itself a benign dentally-based tumor but locally invasive with a high risk of recurrence much like one can consider basal cell carcinoma of the skin. In vitro model systems for studying ameloblastoma are limited due to the fact that these cells grow poorly and begin to enter senescence early. It has been stated that researchers have reported the construction of an ameloblastoma cell line, AM-1, by exposing the cells to human papillomavirus 16 (HPV16) but these cells gain the potential for transformation [24].

Another investigation [25] has outlined that exogenously stably transfected hTERT used to prevent telomere shorterning in fact can immortalize primary human cells without showing cancerous phenotypic properties. In another example, immortalization of pleomorphic adenoma has been obtained via stable hTERT transfection [26]. This has allowed propagation of this cell type in culture without otherwise altering its phenotype. Contact inhibition is altered by immortalization with oncogenic factors such as SV40 and HPVE6/E7 but not so with hTERT.

Many cell lines have been permanently established in this fashion such as fibroblastic, endothelial, mammary and esophageal epithelial and osteoblastic. The ATCC (American Type Culture Collection) repository carries many hTERT immortalized cell lines and this makes a statement in itself as to the success and stability of this procedure. It appears then that genetic changes may be readily manufactured in harvested cells of varying types and lineages to produce an immortalnonsenescing phenotype.

Use of hTERT transformation carries many research advantages for medical research in itself. For example, with the pancreas [27] cells expressing the neuronal stem cell marker nestin are present yet the role for these cells has not been clear. An established immortal pancreatic cell line (as judged by over 150 proliferative cell doublings) by transfection with hTERT demonstrated cells which were positive for the expression of nestin (at both the mRNA and protein levels) and were found to be free of cancer-associated changes. Cells are diploid and express wild type p16(INK4a), p53, and K-Ras. An established line of normal human cells representing this nestin-positive celltype are vital to understanding the importance of nestin expression in the pancreas. Therefore such genetic manipulation to harness the 'immortal side' of cancer holds significant benefit for medicine and will be explored in this article.

\section{Methods: Technologies-the age of the 'shuttle'}

In science, conceptual ideas and advances are oftentimes paralleled by advances in technique and technology. The field of cellular bioengineering is by no-means different in this respect and perhaps epitomizes technological innovation. Although the Atlantis space shuttle unfortunately represents the end of the era of the space shuttle program by the American National Aeronautics and Space Administration (NASA) the era of the 'Shuttle' is not truly over. I refer to shuttle-vector genetics technologies. In this regard, established vectors such as pECV25 are highly useful (ATCC \#77187). This vector is an Epstein-Barr virus derived cDNA shuttle expression vector that replicates episomally in mammalian cells and can use E. coli and mammalian cells as hosts. This plasmid expression vector can be maintained in human cells at a copy number of 25-50 and shows a high transfection efficiency and can be introduced as supercoiled DNA.

In Figure $1 \mathrm{I}$ outline a scheme that can be used to isolate and characterize novel proteins involved in cancer cell therapy-resistance. cDNA inserts in the shuttle vector are efficiently expressed under the RSV promoter and a rabbit beta globin intron with polyadenylation signal. Rescue of the episomal DNA from transfected cells can be readily achieved [28]. Features such as hygromycin selection for transfectants also simplifies working with such a plasmid. This vector has been tried and tested previously as to demonstrate its overall utility $[29,30]$. The scheme (Figure 1) is in itself useful in delineating factors that cancer uses to promote survival and analysis of these can form the basis for survival pathways under physiological conditions as already outlined above with regards my 'prosurvival' model [6]. The scheme can be modified in order to examine those factors that pertain to producing cellular immortality (Figure 2 and Figure 3).

In this respect, cancer can be used to generate a cDNA expression library in pECV25 that can be used to transfect mortal or scenescent cell lines to immortality as measured by population doublings -PDLgreater than 50 but maintaining otherwise normal cellular phenotypic characteristics. These derived stable transfectants can be used to determine the recombinant plasmid(s) responsible by Southern dot blotting. The inserts in the positive recombinants can be analysed to determine their sequence and thereby their identities. Novel factors may be found relating to immortalization that can be of use to generate banks of cell lines representing genetically modified normal cells for implantation (see discussion). Such factors as hTERT that drive immortalization pathways can be discovered by the scheme outlined in Figure 2. Factors such as p16 that are mutated or deleted in the pathway to cellular immortalization [7] could be revealed by following the shuttle scheme as shown in Figure 3.

\section{Discussion}

Much research is currently being performed in order to examine the beneficial effects of stem cell therapy with the end-result of organ regeneration. For example, patients with degenerative osteoarthritis may be able to benefit from autologous mesenchymal stem cell infusion [31]. Bone and cartilaginous tissue formation was evident in cases managed by these means and may offer treatment hopes for individuals with degenerative hip and knee disease secondary to osteoarthritis. The minimally invasive properties of this approach are to be emphasized contrary to overt surgery which is the standard of care today.

Another pressing area for research lies with healing wounds where there is oftentimes the need for research in order to understand how better to manage the wound that continuously breaks down/ This is particularly of relevance to diabetics. A study has shown that mesenchymal stem cells harvested from adipose tissues may offer assistance in this regard [32]. It was noted in this work that adipose 
Citation: Loudon JA (2011) Harvesting the Benefits of Understanding Cancer with the Use of 'Shuttle' Era Technology- Cellular Survival Strategies and Tissue Engineering Via Grafting of Genetically Immortalized Autologous Cells. J Bioanal Biomed S5: 001. doi:10.4172/1948-593X. S5-001

Page 4 of 10

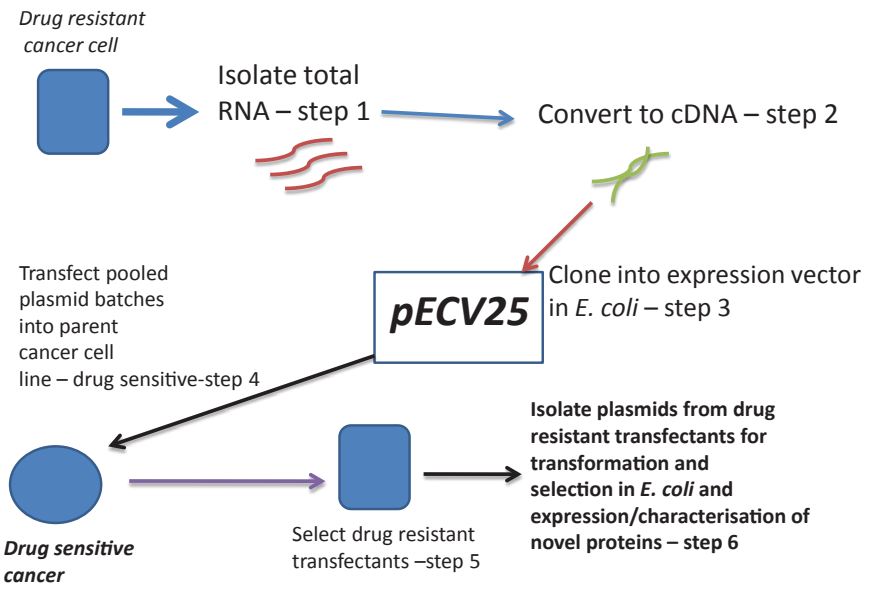

Figure 1: Novel 'shuttle' analysis for determination of cancer therapyresistance factors. In this scheme total RNA is extracted from cancer cells that are therapy resistant. This is then converted into cDNA and cloned into the shuttle expression vector, pECV25 (see text). Plasmids are isolated from individual bacterial clones and pooled into groups of 50 and used to transiently transfect the parent therapy-sensitive cancer cell line. Individual therapyresistant transfectants are selected and plasmid DNA extracted. This can then be transformed back into $E$. coli and DNA from selected clones repeat transfected into parent therapy-sensitive cancer cell lines in order to establish unique transfectants. Alternatively, plasmid DNAs extracted from the first-round of transfection into the parent cancer cells can be restriction digested and nick labelled and dot-blot hydridized against the E. coli colonies enabling unique inserts to be delineated - only after firstly hybridizing against excess unlabelled vector DNA. These clones can be confirmed as carrying therapy-resistance encoding sequences by the second round of transfection into the parent cell line. Suitably isolated plasmid clones can be analyzed to determine the nature of the insert and proteins can be expressed and function assessed.

tissue derived mesenchymal stem cells were able to assist in wound healing in $\mathrm{BALB} / \mathrm{c}$ nude mice. Notably however, differentiated human dermal fibroblasts were more effective in this respect. This is probably due to the already programmed nature of the fibroblast whereas stem cells require to be firstly directed to differentiate along the appropriate lineage.

Recently, it has been shown that human periodontal ligament stem cells are capable of supporting neuronal cell differentiation [33]. The periodontal ligament stem cell line produced neural crest cell markers such as nestin and SOX10 as well as mesenchymal stem cell markers such as CD13 and CD105 and, in addition, nerve growth factor. Therefore, periodontal ligament stem cells are capable of providing for peripheral nerve regeneration in the periodontium and may be adapted to behave in a similar fashion elsewhere in the body to aid repair of peripheral nerve injuries. Neural stem cells are capable of axonal regeneration [34] and promote neural repair in damaged peripheral nerves and allow synaptic connections to be developed between differentiated implanted neural stem cells and the regenerating peripheral neural tissues. Ideally this could assist in reversing muscular atrophy secondary to nerve injuries.

A mechanism is needed to repair damaged cardiac tissue after ischemic disease. It has been noted that certain problems are posed by stem cells being implanted into the ischemic diseased heart due to their limited cell survival [35]. In that study it was suggested that biomaterials could assist in the implantation process and subsequent survival of the stem cells in the heart. By the use of 'novel smart materials' an altered cardiac environment could be created leading to a regenerative heart. It has been found that the benefits of stem cells in cardiac repair and regeneration appears to curiously not lie inherently with the stem cells themselves but with a 'paracrine' effect that these cells have on surrounding host tissue [36]. Understanding this principle may aid in appreciating a means of how to assist in regeneration of cardiac tissue. Indeed it has been said that two mechanisms essentially can be employed in this respect - proliferation of terminally differentiated cells and differentiation of resident cardiac stem cells [37]. The concept of reawakening the regenerative capacity of the adult heart was raised and indeed this is the point of my present review - to outline a means by which cells differentiated along a particular lineage may be able to be genetically modified to grow and divide in an ongoing manner. Doubtlessly this would be of great assistance in replacing large zonal areas of damaged cardiomyocytes within the heart. Having said this, recently mouse bone marrow mesenchymal stem cells have been experimentally transplanted into an acute damage heart model [38]. Interestingly, primary mesenchymal stem cells fared much better at cardiac regeneration than passaged stem cells. It appears that a predictable and reproducible technique is required that is less methodsensitive. That is the aim of the present review with regards geneticallymodified ex-vivo autologous cells for implantation.

One observation that deserves attention is that cardiomyocytes may enter the cell cycle and proliferate and so are not permanently postmitotic [39]. Possibly a reservoir of resident cardiac stem cells is

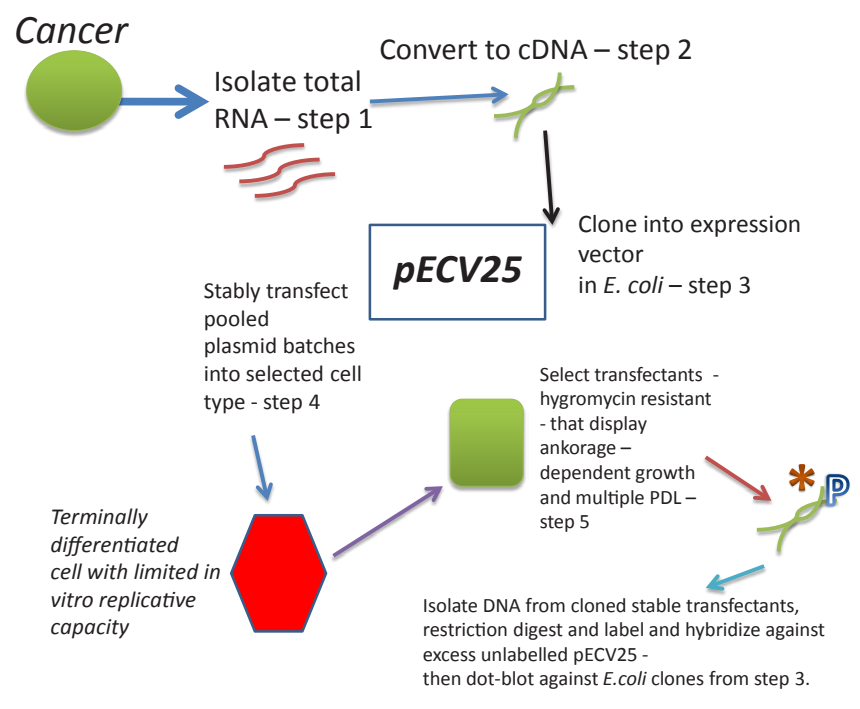

Figure 2: Screening mechanism for isolation of genes involved in determining senescence bypass via 'shuttle' expression vector. In this scheme total RNA is extracted from cancer cells. This is then converted into cDNA and cloned into the shuttle expression vector, pECV25 (see text). Plasmids are isolated from individual bacterial clones and pooled into groups of 50 and used to stably transfect selected terminally differentiated cells with limited in vitro replicative capacity. Individual transfectants are selected that are hygromycin resistant and display ankorage-dependent growth and multiple PDL (see text). DNA is isolated and restriction digested and labelled and hydridized against excess unlabelled vector DNA. Resultant labelled DNA is then used to dot-blot hydridize against $E$.coli clones from step 3 enabling unique inserts to be delineated. Control hydridization against vector alone should be negative. These clones can be confirmed as carrying immortalising genes by a second round of transfection into senescing cell line. Suitably isolated plasmid clones can be analyzed to determine the nature of the insert and proteins can be expressed and function assessed. 
Citation: Loudon JA (2011) Harvesting the Benefits of Understanding Cancer with the Use of 'Shuttle' Era Technology- Cellular Survival Strategies and Tissue Engineering Via Grafting of Genetically Immortalized Autologous Cells. J Bioanal Biomed S5: 001. doi:10.4172/1948-593X. S5-001

Page 5 of 10

present that inherently express telomerase may form a replenishing source of cells as I have previously noted [6,40]. The recently noted 'mircrine' mechanism for cardiac cell regeneration from cardiac stem cells also deserves attention [41]. microRNA-499 favours differentiation of cardiac stem cells to functional cardiomyocytes. Otherwise cardiac stem cells delivered to damaged heart fail to differentiate into fully fledged cardiomyocytes. This interaction of cardiac stem cells with postmitotic myocytes yields functional cardiomyocyte differentiation.

Having said all of the above investigations have been prompted to look at means to improve by genetic means implanted cells for a greater predictable outcome. The main point here is, as has been stated of late [42], that stem cells have potential to differentiate into a variety of cell types. This holds immense value for medicine and total realization of this potential depends on genetic manipulation of stem cells. Gene therapy in terms of a gain/loss of function are very valuable for stem cell manipulations. Having said that, the authors commented that a significant problem lies with the fact that the current status of vectors is still not advanced sufficiently to be efficiently applied to stem cell therapies.

Despite such drawbacks of a technical nature, a recent review has come out in support of the relative benefits of genetically modified mesenchymal stem cells in pancreatic islet transplantation [43]. Also, genetic modification of mesenchymal stem cells for cardiac implantation ex vivo to improve their uptake and survival have been outlined [44]. One means to genetically modify and therefore improve stem cell functionality is via human telomerase (hTERT) modification of human mesenchymal stem cells [45]. Immortalization of human

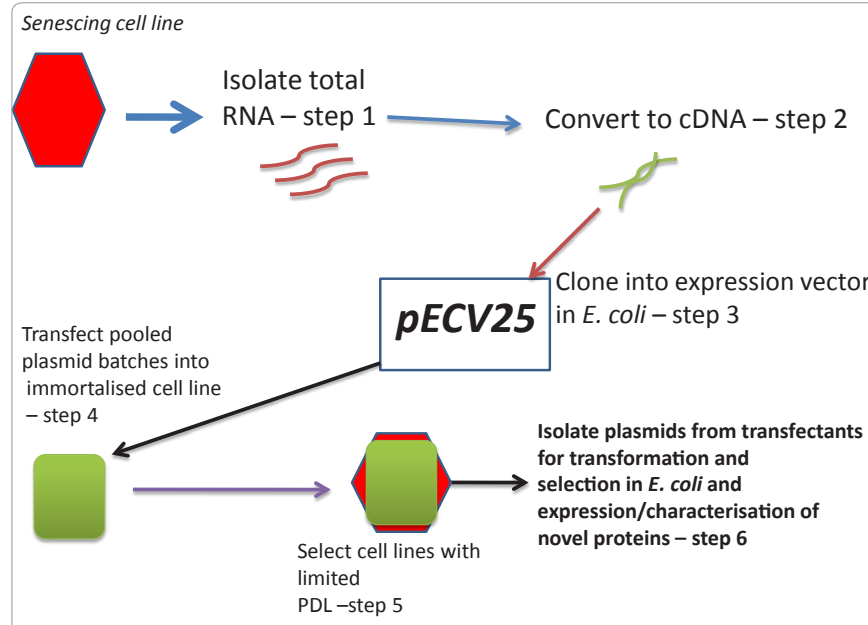

Figure 3: Novel 'shuttle' analysis for determination of factors mutated or deleted in immortalised cell lines. In this scheme total RNA is extracted from senescing cell lines. This is then converted into cDNA and cloned into the shuttle expression vector, pECV25 (see text). Plasmids are isolated from individual bacterial clones and pooled into groups of 50 and used to transiently transfect a selected immortalised cell line. Individual transfectants demonstrating limiting PDL are selected and plasmid DNA extracted. This can then be transformed back into $E$. coli and DNA from selected clones repeat transfected into immortalised cells in order to establish unique transfectants. Alternatively, plasmid DNAs extracted from the first-round of transfection into the immortalised cells can be nick labelled and dot-blot hydridized against the $E$. coli colonies enabling unique inserts to be delineated - after firstly hybridizing against excess unlabelled vector. These clones can be confirmed as carrying sequences that disable cellular immortalisation by the second round of transfection into an immortal cell line. Suitably isolated plasmid clones can be analyzed to determine the nature of the insert and proteins can be expressed and function assessed. bone marrow derived mesenchymal stem cells via retrovirally delivered human telomerase reverse transcriptase cDNA has been achieved [45] One cell line (YKNK-12) was found to be capable of differentiation into adipocytes/osteoblasts/chondrocytes. Osteocyte-differentiated YKNK12 cells were able to regenerate calvarial bone in mice successfully with nil side effects. Appropriate biomarkers for osteogenesis were produced by this cell line. This result clearly shows the benefits of immortalization of stem cells that may allow unlimited supply of such cells for differentiation with subsequent healing capabilities.

Neural stem cells have been transfected with glial cell-line derived neurotrophic factor or brain-derived neurotrophic factor. Artificial conduits of poly(D,L-lactide) seeded with transfected neural stem cells were relatively efficiently capable of sciatic nerve regeneration as assessed functionally [46].

Bone marrow-derived mesenchymal stem cells have been transduced with scleraxis - a transcription factor that is considered to direct tendon development during embryogenesis [47]. Adenoviral scleraxis gene transfection of stem cells from the bone marrow were able to demonstrate improved tendon/bone interfacial healing at early time points [47]. This has significant relevance to the healing of tendon tears and to help in preventing breakdown of same.

Adipose-derived stem cells transfected with insulin-like growth factor-1 grown in chitosan/gelatin scaffolds demonstrated improved chondrogenesis [48]. Recently too it has been shown that there is a flexibility that can be gained by genetic modification of stem cells to yield pluripotency. Mouse neural stem cells can be programmed into induced pluripotent stem cells via expression of Oct4, Sox2, Klf4, c-Myc. These cells can be maintained in culture for many passages ready as use for implantation for tissue regeneration strategies [49].

\section{Downsides of stem cell therapy}

As noted earlier terminally differentiated cells fare better than stem cells in regards wound closure (32). Also problems occur with respect to limited stem cell survival upon their implantation into areas of damaged heart tissue due limiting survival as outlined above (35). Curiously too, stem cell manipulations are technique sensitive in that primary harvested mesenchymal stem cells are better equipped to be employed in cardiac regeneration than stem cells that have been passaged ex vivo as described in the previous section [38]. These negative points serve to indicate that working with stem cells is by no means predictable or technically straightforward.

In my present article, the technology and approach that I choose avoids the use of stem cell therapies and selects cells that are already terminally differentiated. This has intrinsic benefits in that there are some fundamental biological concerns with the use of stem cells or genetically modified stem cells. For example, as it has been pointed out of late [50], the cell of origin of liposarcoma is most likely a pluripotent mesenchymal stem cell. Stem cells therefore have a certain potential to transform. In this respect, the concept of the cancer stem cell must be borne in mind and indeed has gained momentum since the original publication outlining the presence of these cell types in regards hematopoietic malignancies [51]. Telomerase may itself also play a key role in the patho-biology of cancer stem cells [52]. Telomerase activation certainly has a significant role to play in malignant transformation of mesenchymal stem cells [53]. This may serve to limit stem cell manipulations in the practise of tissue engineering and regenerative 
Citation: Loudon JA (2011) Harvesting the Benefits of Understanding Cancer with the Use of 'Shuttle' Era Technology- Cellular Survival Strategies and Tissue Engineering Via Grafting of Genetically Immortalized Autologous Cells. J Bioanal Biomed S5: 001. doi:10.4172/1948-593X. S5-001

Page 6 of 10

medicine. Also, in regards telomerase, cancer progenitor cells express a high level of this factor and are implied to result in treatment resistance and cancer spread $[6,54]$.

Therefore, genetic modification of stem cells is potentially a risky endeavor indeed. Predictable and reliable stable immortalized cells of variously differentiated lineages is required and is the proposition I emphasize in this present review.

\section{Presently proposed genetic ex vivo modification of differentiated cells}

In the above section 'the immortality principle' I outline the situation whereby cells may be immortalized without necessarily causing their malignant transformation - even cells whose origin is that of a benign neoplasm [24-26].

In support of this concept, an interesting study has been outlined that utilized immortalized human vocal fold fibroblasts transduced by hTERT. Appropriate cell-specific connective tissue markers were demonstrated in stable long term cell culture [55]. The authors stated that this now represented a novel and very useful approach to regenerative strategies for the human larynx. Notably, no stem cells were used.

Adenoviral transfer of hTERT into ischemic wounds has been demonstrated to significantly aid their repair [56]. No side effect such as immune response was seen and this result has implications with respect to healing wounds particularly for the older debilitated patient. Improved longer term survival of associated dermal fibrobasts was noted.

A further study is of significance [57]. In this research, the point is made that telomerase expression alone is insufficient to enable certain cell types to evade senescence, much as was indicated in the study quoted above [7]. Such cells, including keratinocytes and mammary epithelial cells, require loss of the pRB/p16INK4a cell cycle control mechanism in addition to hTERT expression to achieve immortality. It was found that introduced hTERT expression immortalized normal mesothelial cells and a premalignant, p16INK4a-negative keratinocyte line. When four keratinocyte strains cultured from normal tissue were transduced to express hTERT, they were incompletely rescued from senescence. Interestingly, it was determined that after reaching the population doubling limit of their parent cell strains, hTERT+ keratinocytes entered a slow growth phase consistent with a senescing phase from which rare, rapidly dividing immortal cells emerged. The point was made that these immortal cells frequently had sustained deletions of the p16 locus or otherwise were deficient in p16INK4a expression. Very importantly, they nevertheless maintained their keratinocyte growth control and differentiated normally in culture and in xenografts. None of the immortalized keratinocytes underwent alteration of their p53 status. A crucial observation was that despite expression of hTERT and loss of p16 function, the immortalised keratinocytes are able to form differentiated epithelia in vitro and in vivo. Immortalised keratinocyte cell lines were able to also show a normal pattern of epidermal differentiation in organotypic culture and in grafts to athymic mice. Thus p16 does not play an essential role in the irreversible growth arrest that precedes normal stratified squamous epithelial differentiation. Cell lines also kept EGF-dependent growth and TPA-sensitive irreversible growth arrest which are normal keratinocyte growth control mechanisms. These particular traits were noted to be consistently found to be lost in advanced squamous cell carcinomas. It was summarized though as a word of caution that given the loss of the p16-mediated growth arrest mechanism and unlimited replicative potential that such cells are predisposed to further changes that may result in malignant transformation. Thus such immortalized keratinocyte cell lines although being of great use for therapeutic means, for example wound healing, merit further investigation.

A further study has examined immortalization of uterine muscle cells from benign tumoral origin to normal myometrial cell lines [58] Human leiomyoma cells grow poorly in culture and senesce early. By hTERT transformation uterine leiomyoma and myometrial cells were immortalized in culture. Analysis of cells for estrogen receptoralpha and progesterone receptor proteins by Western blotting showed similar expression of these proteins between the immortalized and parental leiomyoma and myometrial cells. The immortalized and parent myometrial and leiomyoma cells expressed the smooth musclespecific cytoskeletal protein alpha-actin and were negative for mutant p53 protein via immunocytochemical staining. The immortalized leiomyoma and myometrial cells showed no anchorage-independent growth, with only a small subpopulation of immortalized leiomyoma cells at a higher passage forming two to three small colonies in soft agar. Very importantly, none of the immortalized cells were tumorigenic in nude mice. Thus no significant phenotypic alteration from the parental cells were noted upon immortalization. This example provides a case model for application of immortalization strategies in future approaches to managing damaged uterine tissue.

Adult myometrial stem cell precursors have been noted recently to promote healing of damaged muscles [59]. However, these cells eventually do in fact enter senescence typical of stem cell physiology. Therefore, a stable immortalized 'bank' of cells as produced above [58] would be invaluable in selected internal organ repair.

Overall therefore, genetic modification of differentiated cells ex vivo appears stable and predictable and merits a significant place in the armamentarium of medical therapies to be developed for the future.

\section{Limitations of the ex vivo genetic modification approach and 'shuttle' detection technology}

The approaches outlined in Figures 1-3 represent an oversimplication in terms of developing a genetic strategy to isolate genes of relevance to this present review. For example, in terms of analyzing genes involved in cancer cell survival and therapy resistance it has already been noted that imatinib treatment for chronic myeloid leukemia $(\mathrm{CML})$ may be in part complicated by a silent BCR-ABL oncogene [60]. Detection of these 'silent' oncogenes would otherwise not be possible by the standard regime presented in Figure 1. Promoter methylation is presumed to be a mechanism in the case of BCR-ABL [60]. Hypermethylation in this case is likely given the high CpG content around the transcription start site for BCR. Proposed treatment of CML patients with the DNA demethylating agent 5-azadeoxycyctidine has been considered to unblock the silenced region that can then be targeted by combined imatinib therapy. This is interesting as clearly unblocking treatment of selected cell lines in Figure 1 can be performed in parallel experiments to 'expose' as it were silenced regions that could be in a some way related to resistance determination. Relatively recently it has been shown that quantitative level promoter methylation distinguishes salivary gland carcinoma ex-pleomorphic adenoma from 
Citation: Loudon JA (2011) Harvesting the Benefits of Understanding Cancer with the Use of 'Shuttle' Era Technology- Cellular Survival Strategies and Tissue Engineering Via Grafting of Genetically Immortalized Autologous Cells. J Bioanal Biomed S5: 001. doi:10.4172/1948-593X. S5-001

Page 7 of 10

the benign pleomorphic adenoma [61]. Hence epigenetic variations are very important in determining the cancer phenotype and ought to be taken into consideration in the present 'shuttle' expression vector scheme.

Other interesting findings relate to pseudogenes. It has been recently revealed that far from being non-required relics pseudogenes may be transcribed into RNA - some in a tissue-specific fashion [62]. These transcripts can result in short interfering RNAs that regulate coding genes via $i$ RNA pathway. Pseudogenes may also act in regulating tumor suppressors and oncogenes by acting as microRNA regulators or 'decoys'. In fact, the pseudogene PTENP1 is biologically active and regulates the levels of the tumor suppressor PTEN. Loss of PTENP1 is seen in cancer [63]. Determination of such pseudogene sequences would be advantageous and modification of the expression strategy with pECV - by transfecting recombinants into a selected cancer cell line and thence selecting for clones demonstrating an inhibited cancer phenotype and analyzing the insert in the shuttle vector may prove necessary in this regard. Certainly the pseudogene story is evolving rapidly and, as an aside, inherently weakens such arguments as used by Avise [64] who describes pseudogenes as 'pervasive architectural flaws' in support of his contention for a 'nonsentient design' of the human genome, in other words, not designed by any external powerful intelligent agent. If anything, recent findings support quite the opposite argument that the genome is highly organized - yet subtle in this respect. As the language of genetics is indeed 'hieroglyphic' and as yet just being unraveled.

Already a word of caution has been made regarding immortalization of human cells for tissue repair [57]. There is no doubt that this approach represents very 'early days' and that longer term follow-up of immortalized cells is very much required. Having said this, it is one feature of the developed 'shuttle' technology in this review to pinpoint further factors that may be involved in promoting the immortal phenotype without inducing transformation. The 'shuttle' technology can aid determining those factors involved in senescence bypass (Figure 2) or those required to be altered to allow cells to reach the immortal phenotype (Figure 3). In other words, the continued search remains for those genetic factors that will enable terminally differentiated cells to stably maintain immortal properties without risk for transformation. This is the ideal goal for regenerative strategies based on this approach.

Thus to conclude this section it can be said that it is an oversimplication to consider such techniques as proposed in this present review as definitive in respect to genome-wide screening. Ultimately, an 'evolution' of the 'shuttle-genetic' approach will necessarily take place.

\section{The novel 'shuttle' design and cancer survival factors}

In this article I have presented a novel way to approach gene characterization for cancer resistance genes as well as genes that are potentially capable of being related to the immortal phenotype of cancer. Figure 1 presents a useful scheme in order to 'shuttle vector' determine those factors that when expressed relate to cancer resistance to therapy. It should be noted that traditionally, cancer resistant cell lines are usually developed from parent cancer therapy-sensitive cells and comparative proteomic and microarray strategies are employed as I have already outlined in a recent review on survival factors $[6,65]$. The effects of various environmental conditions oftentimes found challenging tumors can also be examined in this fashion. For example, recently it has been shown that hypoxia leads to beta-catenin downregulation involving a pathway using p53-dependent activation of Siah1 - the human homolog of Drosophila seven-in-absentia [66]. Clearly, growing the cells under a hypoxic challenge and thence isolating RNA and transfecting via shuttle vector into cells not grown under hypoxic challenge to determine the resultant effects upon beta-catenin status could reveal paths in control of this key factor in hypoxia. Also, determining to what extent factors that may lead to cell survival under hypoxia may be assessed by transfection of recombinant shuttle vectors bearing inserts derived from cell lines accommodated to hypoxia into cells grown under regular normoxic conditions thence challenged with hypoxia may isolate factors involved in cellular survival under harsh hypoxic conditions. There may well be several uses of shuttle bioengineering in respect to this application in determining cancer cell resistance to environmental effects overall.

Overall, adverse external environment has the effect of cancer mounting a defense strategy, much like the classical physiological 'fight or flight' response that I have described [6]. The subject of my recent review [6] has been that of probing for survival factors that cancer employs to determine its resistance to challenging environmental conditions. The in vivo model of the heart was considered adequate and well suited in this respect.

Very recently, basal cell luminal mammary gland carcinoma cells demonstrate an increased rate of metastasis in response to antiangiogenesis therapies [67]. It was noted that conditioned medium from hypoxic cells induced migration in cells grown under normoxia. As stated above, the scheme employed to analyze factors from cancer cells responding to hypoxia via the 'shuttle' expression vector methodology could well be used in this instance as well. This would assist in characterizing those factors determining migratory activity related to cancer spread - part of cancer's defensive 'flight' response.

A further recent article has indicated that hypoxia-induced alternatively spliced variants have roles to play in cellular survival. The message here comes 'from the heart' so to speak where to date an unrecognized splice variant of the mitochondrial apoptosis-related gene Bnip3 - viz: Bnip3deltaex3 associates with Bnip3 and downregulates its activity thus protecting against apoptosis under hypoxic external challenging conditions [68]. This is a very important observation and has major potential ramifications with respect to cancer-as according to my prosurvival model [6] there is a close mechanistic tie between cancer and heart under external challenge. The effect of Bnip3deltaex3 splice variant may well be very important in cancer's survival strategy. Alternatively spliced variants could well be detected via the 'shuttle' expression vector approach described in this review and ought to be considered as a further nuance in terms of cancer's elaborate pattern of response to therapy resistance and survival overall.

\section{Conclusion}

Many advantages come from immortalizing already terminally differentiated cells for tissue engineering. In order to achieve this, improved understanding of the genetics behind cellular immortalization is needed. My article addresses these points both from a conceptual as well as technical viewpoint through harnessing a number of the useful properties cancer displays.

Cells that are already differentiated possess the ready-made fabric as it were for tissue and/or organ regeneration without recourse to cell 
Citation: Loudon JA (2011) Harvesting the Benefits of Understanding Cancer with the Use of 'Shuttle' Era Technology- Cellular Survival Strategies and Tissue Engineering Via Grafting of Genetically Immortalized Autologous Cells. J Bioanal Biomed S5: 001. doi:10.4172/1948-593X. S5-001

Page 8 of 10

differentiation along a particular pathway. This enables for a seemingly more predictable pattern of functional cellular replacement.

Having said this, autologous adult stem cell use for introducing genes into wounds and defects may well have a bright future [69]. No doubt, all the approaches outlined in my present review shall have a place of importance in organ regeneration at some point in the future.

Another key feature of this review, and one that runs as a theme throughout my analysis is that of cellular survival. The final common denominator in biology whether it is physiology or pathology is cellular survival. Cancer shows us how this may be achieved as this process represents the 'ultimate' in cell survival accomplishment. The shuttle vector concept developed here in this review seeks to further establish important survival paths that can be used not just in terms of tissue regeneration and sustenance but also to modify and target in cancer for future biotherapeutics. The overall scheme for shuttle era technologies may be modified further to examine the contribution individual survival pathways may have in cancer growth and invasion. For example, the vector presented in this review could be engineered to release silencing RNA for gene knockdown (small interfering RNA - siRNA). Introduction of such survival pathway factor silencing RNA recombinant constructs into cancer cells may produce significant growth retarding properties. Screening for novel survival factor biotargets may well be facilitated by the use of such novel vectors. Overall, uses are many for shuttle-designed cloning technologies.

Interestingly, in regards survival pathways, the prosurvival factor Peroxisome Proliferative Activated Receptor gamma (PPARgamma) has been discussed recently by myself [6]. This factor has been shown to be needed for generation of healthy hair follicular structures [70]. Targeted deletion of PPARgamma in follicular stem cells removes the functioning hair follicular unit. This result shows that PPARgamma as a prosurvival factor [6] is required for hair follicle organ development and as such demonstrates that prosurvival factors are key in the process of tissue/organ regeneration. This is a key statement I make in this review whereby the search for survival proteins as discussed in my prosurvival model will lend important information towards effective organ regeneration ultimately.

Regulation of embryonic stem cells by polycomb protein groupdependent trimethylation on histone H3K27 controls their identity and thus tissue development [71]. The methyltransferases involved viz: Ezh1/2 are required for hair follicular development. Curiously though, epidermis does not have a strict requirement in regards Ezh1/2 for healing [71]. In Ezh1/2 null background the p16 gene is fully activated in hair follicular structures but only partially so in epidermal progenitors [71]. Silencing of p16 in hair follicules in Ezh1/2 null background restores proliferation of the follicular unit cells - at least in vitro. Clearly p16 is providing a 'brake' and this is consistent with the information already discussed in this review in that p16 is a key player in determining cell proliferation/immortal transformation. Having said this, as this study of late shows [71] it is coming to light that many other modifying factors such as histone methyltransferases are also involved in maintaining ongoing cellular proliferation. My present review is focused on determining via novel alternative technologies the identity of such additional key factors (see Figure 2 and Figure 3).

Finally, harnessing an improved understanding of cancer and how it copes and adapts to its environment and how it has evolved into the efficient self-perpetuating parasite that it has may well have many sidebenefits for medicine. Tackling these points and questions will certainly be a major challenge and effort for the personalized medicine strategies of the future to consider and to take on to develop into productive benefits for medicine.

\section{Acknowledgement}

I thank Dr George Al-Horani, principal, Wetherill Park Medical Centre, for his support and encouragement during the period of preparing this review.

\section{References}

1. Raya-Rivera A, Esquiliano DR, Yoo JJ, Lopez-Bayghen E, Soker S, et al. (2011) Tissue-engineered autologous urethras for patients who need reconstruction an observational study. Lancet 377: 1175-1182.

2. Atala A, Bauer SB, Soker S, Yoo JJ, Retik AB (2006) Tissue-engineered autologous bladders for patients needing cystoplasty. Lancet 367: 1241-1246.

3. Petrovic N, Black TA, Fabian JR, Kane C, Jones CA, et al. (1996) Role of proximal promoter elements in regulation of renin gene transcription. $\mathrm{J}$ Bio Chem 271: 22499-22505

4. Mascarenhas AK, Allen CM, Loudon J (2001) The association between Viadent use and oral leukoplakia. Epidemiology 12: 741-743.

5. Loudon JA (2010) Cancer: a novel "no-nonsense" approach to treatment Oncol Res 19: 55-66

6. Loudon JA (2011) A novel pro-survival model for cancer under environmenta challenge: the "heart-felf" message for therapeutic intervention. Oncol Res In Press.

7. Muntoni A, Fleming J, Gordon KE, Hunter K, McGregor F, et al. (2003) Senescing oral dysplasias are not immortalized by ectopic expression of hTERT alone without other molecular changes, such as loss of INK4A and or retinoic acid receptor-beta: but p53 mutations are not necessarily required. Oncogene 22: 7804-7808.

8. Chung YM, Yoo YD, Park JK, Kim YT, Kim HJ (2001) Increased expression of peroxiredoxin II confers resistance to cisplatin. Anticancer Res 21: 1129-1133.

9. Nagy N, Malik G, Fisher AB, Das DK (2006) Targeted disruption of peroxiredoxin 6 gene renders the heart vulnerable to ischemia-reperfusion injury. Am J Physiol Heart Circ Physiol 291: H2636-22640.

10. Nista A, Leonetti C, Bernardini G, Mattioni M, Santoni A (1997) Functional role of alpha4beta1 and alpha5beta1 integrin fibronectin receptors expressed on adriamycin-resistant MCF-7 human mammary carcinoma cells. Int J Cancer 72 133-141.

11. Dallabrida SM, Ismail N, Oberle JR, Himes BE, Rupnick MA (2005) Angiopoietin-1 promotes cardiac and skeletal myocyte survival through integrins. Circ Res 96: e8-24.

12. Toth A, Nickson P, Mandl A, Bannister ML, Toth K, et al. (2007) Endoplasmic reticulum stress as a novel therapeutic target in heart diseases. Cardiovasc Hematol Disord Drug Targets 7: 205-218.

13. Petrovski G, Das S, Juhasz B, Kertesz A, Tosaki A, et al. (2011) Cardioprotection by endoplasmic reticulum stress-induced autophagy. Antioxid Redox Signal 14 2191-2200.

14. Ranganathan AC, Zhang L, Adam AP, Aguirre-Ghiso JA (2006) Functiona coupling of p38-induced up-regulation of BiP and activation of RNA-dependent protein kinase-like endoplasmic reticulum kinase to drug resistance of dorman carcinoma cells. Cancer Res 66: 1702-1711.

15. Di Michele M, Marcone S, Cicchillitti L, Della Corte A, Ferlini C, et al. (2010) Glycoproteomics of paclitaxel resistance in human epithelial ovarian cancer cel lines: towards the identification of putative biomarkers. J Proteomics 73: 879 898

16. Stewart JJ, White JT, Yan X, Collins S, Drescher CW, et al. (2006) Proteins associated with Cisplatin resistance in ovarian cancer cells identified by quantitative proteomic technology and integrated with mRNA expression levels. Mol Cell Proteomics 5: 433-443. 
Citation: Loudon JA (2011) Harvesting the Benefits of Understanding Cancer with the Use of 'Shuttle' Era Technology- Cellular Survival Strategies and Tissue Engineering Via Grafting of Genetically Immortalized Autologous Cells. J Bioanal Biomed S5: 001. doi:10.4172/1948-593X. S5-001

17. Strohm C, Barancík M, von Bruehl M, Strniskova M, Ullmann C et al. (2002) Transcription inhibitor actinomycin-D abolishes the cardioprotective effect of ischemic reconditioning. Cardiovasc Res 55: 602-618.

18. Sakamoto K, Nakahara T, Ishii K (2011) Rho-Rho kinase pathway is involved in the protective effect of early ischemic preconditioning in the rat heart. Biol Pharm Bull 34: 156-159.

19. Zhai GG, Malhotra R, Delaney M, Latham D, Nestler U, et al. (2006) Radiation enhances the invasive potential of primary glioblastoma cells via activation of the Rho signaling pathway. J Neurooncol 76: 227-237.

20. Skvortsova I, Skvortsov S, Stasyk T, Raju U, Popper BA, et al. (2008) Intracellular signaling pathways regulating radioresistance of human prostate carcinoma cells. Proteomics 8: 4521-4533.

21. Kleinridders A, Pogoda HM, Irlenbusch S, Smyth N, Koncz C, et al. (2009) PLRG1 is an essential regulator of cell proliferation and apoptosis during vertebrate development and tissue homeostasis. Mol Cell Biol 29: 3173-3185.

22. Wu CC, Chen HC, Chen SJ, Liu HP, Hsieh YY, et al. (2008) Identification of collapsin response mediator protein-2 as a potential marker of colorectal carcinoma by comparative analysis of cancer cell secretomes. Proteomics 8 : 316-332

23. Hamajima N, Matsuda K, Sakata S, Tamaki N, Sasaki M, et al. (1996) A nove gene family defined by human dihydropyrimidinase and three related proteins with differential tissue distribution. Gene 180: 157-163.

24. Tao Q, Lv B, Qiao B, Zheng CQ, Chen ZF (2009) Immortalization of ameloblastoma cells via reactivation of telomerase function: Phenotypic and molecular characteristics. Oral Oncol 45: e239-244.

25. Lee KM, Choi KH, Ouellette MM (2004) Use of exogenous hTERT to immortalize primary human cells. Cytotechnology 45: 33-38.

26. Kitagawa M, Ogawa I, Shima K, Hashimoto S, Kudo Y, et al. (2007) Immortalization and characterization of pleomorphic adenoma cells by transfection with the hTERT gene. Int J Oncol 31: 339-344.

27. Lee KM, Nguyen C, Ulrich AB, Pour PM, Ouellette MM (2003) Immortalization with telomerase of the Nestin-positive cells of the human pancreas. Biochem Biophys Res Commun 301: 1038-1044.

28. Belt PB, Groeneveld H, Teubel WJ, van de Putte P, Backendorf C (1989) Construction and properties of an Epstein-Barr-virus-derived cDNA expression vector for human cells. Gene 84: 407-417.

29. Belt PB, Jongmans W, de Wit J, Hoeijmakers JH, van de Putte P, et al. (1991) Efficient cDNA cloning by direct phenotypic correction of a mutant human cell line (HPRT-) using an Epstein-Barr virus-derived cDNA expression vector. Nucleic Acids Res 19: 4861-4866.

30. Belt PB, van Oosterwijk MF, Odijk H, Hoeijmakers JH, Backendorf C (1991) Induction of a mutant phenotype in human repair proficient cells after overexpression of a mutated human DNA repair gene. Nucleic Acids Res 19 : 5633-5637.

31. Pak J (2011) Regeneration of human bones in hip osteonecrosis and human cartilage in knee osteoarthritis with autologous adipose-tissue-derived stem cells: a case series. J Med Case Reports 5: 296

32. Lee SH, Lee JH, Cho KH (2011) Effects of Human Adipose-derived Stem Cells on Cutaneous Wound Healing in Nude Mice. Ann Dermatol 23: 150-155.

33. Tomokiyo A, Maeda H, Fujii S, Monnouchi S, Wada N, et al. (2011) A multipotent clonal human periodontal ligament cell line with neural crest cell phenotypes promotes neurocytic differentiation, migration, and survival. J Cell Physiol doi: 10.1002/jcp.22933.

34. Gu SH, Xu WD, Xu L, Li XK, Ochiya T, et al. (2010) Regenerated host axons form synapses with neurons derived from neural stem cells transplanted into peripheral nerves. J Int Med Res 38: 1721-1729.

35. Pereira MJ, Carvalho IF, Karp JM, Ferreira LS (2011) Sensing the Cardiac Environment: Exploiting Cues for Regeneration. J Cardiovasc Transl Res 4: $616-630$

36. Gharaibeh B, Lavasani M, Cummins JH, Huard J (2011) Terminal differentiation is not a major determinant for the success of stem cell therapy - cross-talk between muscle-derived stem cells and host cells. Stem Cell Res Ther 2: 31.
37. Kühl SJ, Kühl M (2011) Improving cardiac regeneration after injury: Are we a step closer? Bioessays 33: 669-673

38. Jin J, Zhao Y, Tan X, Guo C, Yang Z, et al. (2011) An improved transplantation strategy for mouse mesenchymal stem cells in an acute myocardial infarction model. PLoS One 6: e21005.

39. De Falco M, Cobellis G, De Luca A (2009) Proliferation of cardiomyocytes: a question unresolved. Front Biosci (Elite Ed) 1: 528-536.

40. Schneider MD (2006) Dual roles of telomerase in cardiac protection and repair Novartis Found Symp 274: 260-267; discussion 267-76.

41. Hosoda T, Zheng H, Cabral-da-Silva M, Sanada F, Ide-Iwata N, et al. (2011) Human cardiac stem cell differentiation is regulated by a mircrine mechanism. Circulation 123: 1287-1296.

42. Adly AS, Aboutabl AE, Ibrahim MS (2011) Modeling of gene therapy fo regenerative cells using intelligent agents. Adv Exp Med Biol 696: 317-325.

43. Wu H, Ye Z, Mahato RI (2011) Genetically Modified Mesenchymal Stem Cells for Improved Islet Transplantation. Mol Pharm 8: 1458-1470.

44. Song H, Song BW, Cha MJ, Choi IG, Hwang KC (2010) Modification of mesenchymal stem cells for cardiac regeneration. Expert Opin Biol Ther 10 309-319.

45. Nakahara H, Misawa H, Hayashi T, Kondo E, Yuasa T, et al. (2009) Bone repair by transplantation of hTERT-immortalized human mesenchymal stem cells in mice. Transplantation 88: 346-353.

46. Fu KY, Dai LG, Chiu IM, Chen JR, Hsu SH (2011) Sciatic nerve regeneration by microporous nerve conduits seeded with glial cell line-derived neurotrophic factor or brain-derived neurotrophic factor gene transfected neural stem cells. Artif Organs 35: 363-372.

47. Gulotta LV, Kovacevic D, Packer JD, Deng XH, Rodeo SA (2011) Bone marrow-derived mesenchymal stem cells transduced with scleraxis improve rotator cuff healing in a rat model. Am J Sports Med 39: 1282-1289.

48. Li J, Zhao Q, Wang E, Zhang C, Wang G, Yuan Q (2011) Dynamic compression of rabbit adipose-derived stem cells transfected with insulin-like growth factor in chitosan/gelatin scaffolds induces chondrogenesis and matrix biosynthesis. J Cell Physiol doi: 10.1002/jcp.22927.

49. Choi HW, Kim JS, Choi S, Jang HJ, Kim MJ, et al. (2011) Neural Stem Cells Achieve and Maintain Pluripotency without Feeder Cells. PLoS One 6: e21367.

50. Steger CM (2011) Primary Liposarcoma of the heart. British Medical Journa Online Case Reports.

51. Bonnet D, Dick JE (1997) Human acute myeloid leukemia is organized as a hierarchy that originates from a primitive hematopoietic cell. Nat Med 3: 730737

52. Bollmann FM (2008) The many faces of telomerase: emerging extratelomeric effects. Bioessays 30: 728-732.

53. Yamaoka E, Hiyama E, Sotomaru Y, Onitake Y, Fukuba I, et al. (2011) Neoplastic transformation by TERT in FGF-2-expanded human mesenchyma stem cells. Int J Oncol 39: 5-11.

54. Xu T, He K, Wang L, Goldkorn A (2011) Prostate tumor cells with cance progenitor properties have high telomerase activity and are rapidly killed by telomerase interference. Prostate 71: 1390-1400.

55. Chen X, Thibeault SL (2009) Novel isolation and biochemical characterization of immortalized fibroblasts for tissue engineering vocal fold lamina propria. Tissue Eng Part C Methods 15: 201-212.

56. Mogford JE, Liu WR, Reid R, Chiu CP, Said H, et al. (2006) Adenoviral human telomerase reverse transcriptase dramatically improves ischemic wound healing without detrimental immune response in an aged rabbit model. Hum Gene Ther 17: 651-660.

57. Dickson MA, Hahn WC, Ino Y, Ronfard V, Wu JY, et al. (2000) Rheinwald JG. Human Keratinocytes That Express hTERT and Also Bypass a p16INK4aEnforced Mechanism That Limits Life Span Become Immortal yet Retain Normal Growth and Differentiation Characteristics. Mol Cell Biol 20: 1436-1447. 
Citation: Loudon JA (2011) Harvesting the Benefits of Understanding Cancer with the Use of 'Shuttle' Era Technology- Cellular Survival Strategies and Tissue Engineering Via Grafting of Genetically Immortalized Autologous Cells. J Bioanal Biomed S5: 001. doi:10.4172/1948-593X. S5-001

Page 10 of 10

58. Carney SA, Tahara H, Swartz CD, Risinger JI, He H, et al. (2002) Immortalization of human uterine leiomyoma and myometrial cell lines after induction of telomerase activity: molecular and phenotypic characteristics. Lab Invest 82: 719-728.

59. Gálvez BG, Martín NS, Salama-Cohen P, Lazcano JJ, Coronado MJ, et al. (2010) An adult myometrial pluripotential precursor that promotes healing of damaged muscular tissues. In Vivo 24: 431-441.

60. Jiang G, Yang F, Li M, Weissbecker K, Price S, et al. (2003) Imatinib (ST1571) provides only limited selectivity for $\mathrm{CML}$ cells and treatment might be complicated by silent BCR-ABL genes. Cancer Biol Ther 2: 103-108.

61. Schache AG, Hall G, Woolgar JA, Nikolaidis G, Triantafyllou A, et al. (2010) Quantitative promoter methylation differentiates carcinoma ex pleomorphic adenoma from pleomorphic salivary adenoma. Br J Cancer 103: 1846-1851.

62. Pink RC, Wicks K, Caley DP, Punch EK, Jacobs L, et al. (2011) Pseudogenes: pseudo-functional or key regulators in health and disease? RNA 17: 792-798.

63. Poliseno L, Salmena L, Zhang J, Carver B, Haveman WJ, et al. (2010) A coding-independent function of gene and pseudogene mRNAs regulates tumour biology. Nature 465: 1033-1038.

64. Avise JC (2010) Colloquium paper: footprints of nonsentient design inside the human genome. Proc Natl Acad Sci USA 107 Suppl 2 :8969-8976.

65. Lee YS, Oh JH, Yoon S, Kwon MS, Song CW, et al. (2010) Differential gene expression profiles of radioresistant non-small-cell lung cancer cell lines established by fractionated irradiation: tumor protein p53-inducible protein 3 confers sensitivity to ionizing radiation. Int J Radiat Oncol Biol Phys 77: 858866 .

66. Wang D, Wang $Y$, Kong $T$, Fan F, Jiang $Y$ (2011) Hypoxia-induced $\beta$-catenin downregulation involves p53-dependent activation of Siah-1. Cancer Sci 102 1322-1328.

67. Voss MJ, Möller MF, Powe DG, Niggemann B, Zänker KS, et al. (2011) Luminal and basal-like breast cancer cells show increased migration induced by hypoxia, mediated by an autocrine mechanism. BMC Cancer 11: 158.

68. Gang H, Hai Y, Dhingra R, Gordon JW, Yurkova N, et al. (2011) A novel hypoxia-inducible spliced variant of mitochondrial death gene Bnip3 promotes survival of ventricular myocytes. Circ Res 108: 1084-1092.

69. Gauglitz GG, Jeschke MG. Combined Gene and Stem Cell Therapy for Cutaneous Wound Healing. Mol Pharm 2011 8: 1471-1479.

70. Karnik P, Tekeste Z, McCormick TS, Gilliam AC, Price VH, et al. (2009) Hair follicle stem cell-specific PPARgamma deletion causes scarring alopecia. $J$ Invest Dermatol 129: 1243-1257.

71. Ezhkova E, Lien WH, Stokes N, Pasolli HA, Silva JM, et al. (2011) EZH1 and $\mathrm{EZH} 2$ cogovern histone H3K27 trimethylation and are essential for hair follicle homeostasis and wound repair. Genes Dev 25: 485-498. 